\title{
Tuberous sclerosis complex in the Western Cape, South Africa: The clinical presentation features
}

\author{
E Kija, ${ }^{1,2}$ MMed; B Schlegel, ${ }^{1}$ FCP; P Samia,,${ }^{1,3}$ MPhil; M Wessels, ${ }^{1}$ MMed; J M Wilmshurst, ${ }^{1}$ MD \\ ${ }^{1}$ Department of Paediatric Neurology, Red Cross War Memorial Children's Hospital, University of Cape Town, South Africa \\ ${ }^{2}$ Department of Paediatrics, School of Medicine, Muhimbili University of Health and Allied Sciences, Dar es Salaam, Tanzania \\ ${ }^{3}$ Department of Paediatrics, Medical College, Aga Khan University, Nairobi, Kenya
}

Corresponding author: J M Wilmshurst (jo.wilmshurst@uct.ac.za)

\begin{abstract}
Tuberous sclerosis complex (TSC) is a genetic neurocutaneous condition, which affects multiple organ systems. This study aimed to determine the presenting features of children with TSC in Cape Town, South Africa. A cross-sectional study was conducted at a TSC clinic, and clinical features at presentation were prospectively collected. Thirty-nine children ( 23 boys; median age 10 (range 1 - 26) years; median diagnosis age 16 ( 0 - 153) months) were recruited. Twenty-one (54\%) children presented with focal seizures. Seven (18\%) children had epileptic spasms. Skin manifestations led to a diagnosis in 13 (33\%) and neuroimaging in 22 (56\%) children. Antenatal screening detected cardiac rhabdomyomas in 3 children. One child had a positive family history. In the paediatric service, TSC diagnosis usually followed neuroimaging, whereas at the neurology service skin manifestations indicated TSC. In conclusion, most children with TSC presented as emergency cases with seizures. Health practitioner awareness of the common TSC clinical signs was lacking, with the diagnosis often delayed.
\end{abstract}

S Afr Med J 2017;107(4):295-298. DOI:10.7196/SAMJ.2017.v107i4.12040

Tuberous sclerosis complex (TSC) is a genetically inherited condition that manifests with benign non-invasive hamartomas in multiple organ systems and is associated with varied clinical presentations. ${ }^{[1,2]}$ The prevalence of TSC worldwide is reported as 1 in 6000 newborns. ${ }^{[1-4]}$ Equivalent data from Africa are lacking. In Africa, the burden of disease is dominated by infectious aetiologies. People with non-communicable disorders, especially of genetic origin, have access to scarce resources and are managed as low-priority cases. This results in disparate outcomes, e.g. in well-resourced settings awareness of TSC is more likely to lead to early diagnosis, whereas in Africa management is reactive, and limited resources and poor awareness lead to delayed diagnosis and intervention.

Red Cross War Memorial Children's Hospital (RCWMCH), Cape Town, South Africa (SA) is unique in that it provides a dedicated TSC service in the paediatric neurology department. The service was established $>15$ years ago and has, during this period, managed 45 affected children. Based on international prevalence figures, this number is less than expected for the region and raises concerns that only a proportion of affected children access the service. The referral pattern and disease demographics of the service were examined to identify remedial factors to improve diagnosis and service delivery.

\section{Objective}

The objective of this study was to document the phenotype of SA children with TSC, identifying clinical features at presentation, which could lead to improvements in diagnosis in our setting.

\section{Methods}

RCWMCH is a university teaching hospital located in the Western Cape, SA. For children with TSC a dedicated multidisciplinary service operates in the paediatric neurology department, in line with international management guidelines. Patients are referred to our hospital by local health practitioners, based on concerns that arise from the complications of TSC. As such, the understanding of referral patterns was considered important to develop interventions to promote earlier identification of affected children, which would facilitate preventive rather than reactive interventions.

All children attending the service between October 2013 and August 2016 were prospectively recruited.

Patient demographics were recorded and compared with international data (Table 1). ${ }^{[5-7]}$ Age at presentation, referral sources and reason for referral were also documented.

Ethical clearance to conduct the study was granted by the Faculty of Health Sciences Research Ethics Committee, University of Cape Town (ref. no. 020/2013). Written consent was obtained from caregivers.

\section{Results}

Over the 33-month period, consent was obtained for 39 children (23 males, 16 females; median 10 (range 1 - 26) years), who fulfilled the international diagnostic criteria for TSC, to be part of the study. From the original cohort attending the service $(n=45), 5$ children had been referred to adult services, and 1 child died because of complications unrelated to his TSC diagnosis.

Sixteen children were of indigenous black African ancestry, 9 were white, 13 of mixed ancestry and 1 was Arabic. Median age at diagnosis was $16(0$ - 153) months (Table 1$)$.

Twenty-one (54\%) children were referred to the general neurology service owing to focal seizures, where TSC was diagnosed. Diagnosis was delayed in four children. Two were mislabelled on neuroimaging, specifically with neurocysticercosis in one child and congenital cytomegalovirus in another. TSC was diagnosed following identification of hypopigmented skin lesions in the former child and after persistent hypertension and identification of renal angiomyolipomas in the latter. A third child suffered recurrent 
Table 1. Demographics of children attending the TSC service from October 2013 to August 2016 compared with those of other similar paediatric cohort studies ${ }^{[5-7]}$

\begin{tabular}{|c|c|c|c|c|}
\hline Variable & Current study, $N=39$ & Yates et al. ${ }^{[5]} N=114$ & $\begin{array}{l}\text { Datta et al. }{ }^{[6]} \\
\text { (infants only), } N=41\end{array}$ & Erol et al.,$^{[7]} N=20$ \\
\hline Male:female & 23:16 & $62: 63$ & $24: 17$ & $10: 10$ \\
\hline Age at presentation (months), median & 16 & 7 & 1.5 & 11 \\
\hline Subependymal giant cell astrocytomas, ${ }^{*} n(\%)$ & $10(26)$ & $5(4)$ & $4(10)$ & $1(5)$ \\
\hline Epilepsy, $n(\%)$ & $34(87)$ & - & - & $17(85)$ \\
\hline Focal epilepsy, $n(\%)$ & $21(54)$ & - & - & $7(35)$ \\
\hline $\begin{array}{l}\text { Epileptic spasms ( } 3 \text { focal epilepsy and } \\
\text { epileptic spasms), } n(\%)\end{array}$ & $7(18)$ & - & - & $7(35)$ \\
\hline Cortical tubers, ${ }^{\star} n(\%)$ & $31(79)$ & $93(89)$ & $35(85)$ & $16(80)$ \\
\hline Subependymal nodules, ${ }^{*} n(\%)$ & $36(92)$ & $76(73)$ & $37(90)$ & $16(80)$ \\
\hline Intellectual disability, $n(\%)$ & $23(59)$ & $75(85)$ & - & $5 / 8(63)$ \\
\hline Angiomyolipoma, ${ }^{*} n(\%)$ & $17(44)$ & $13(12)$ & $6(15)$ & $1(5)$ \\
\hline Cardiac rhabdomyoma, ${ }^{\star} n(\%)$ & $8(20)$ & $51(58)$ & $35(85)$ & - \\
\hline Facial angiofibroma, ${ }^{\star} n(\%)$ & $19(49)$ & $24(29)$ & $24(29)$ & $8(40)$ \\
\hline$>3$ hypomelanotic macules, ${ }^{*} n(\%)$ & $27(69)$ & $80(65)$ & $21(51)$ & $17(85)$ \\
\hline Shagreen patch, ${ }^{\star} n(\%)$ & $10(28)$ & $30(24)$ & $30(73)$ & $2(10)$ \\
\hline Ungual and periungual fibroma, ${ }^{*} n(\%)$ & $1(0.02)$ & $3(2)$ & - & - \\
\hline
\end{tabular}

pancreatitis, and her skin lesions were initially thought to be due to a fungal infection. She eventually underwent a brain magnetic resonance imaging (MRI) scan and the skin lesions were re-examined. A fourth child was resident in a rural part of the country. His learning and behavioural problems were well known to his caregivers, but he attended the health service only when he was 12 years and 9 months of age, with poorly controlled seizures.

Seven (18\%) children had epileptic spasms, 4 at presentation, and 3 with focal seizures later developed spasms. The median lag time from onset of epileptic spasms to diagnosis and intervention was 3 (2 - 8) months. Skin manifestations led to referral in 13 (33\%) children, who were already being managed for seizures. Neuroimaging as part of routine screening for seizures resulted in confirmation of TSC in a further $22(56 \%)$ children. Three children with cardiac rhabdomyomas were identified antenatally, and 1 child with a positive family history was also diagnosed antenatally.

Most children were referred to the general neurology service as emergency cases with prolonged convulsive seizures, and a smaller proportion were referred from the medical outpatient service with brief seizures. The hospital location of the child at the time of diagnosis was distributed as follows: medical outpatients $(n=9)$, paediatrics $(n=9)$, neurology $(n=15)$, antenatal obstetric service $(n=3)$, and 1 each for genetics, gastroenterology and dermatology services. The key marker that led to the diagnosis of TSC was predominantly based on neuroimaging findings after routine investigation for seizures in children managed in the medical outpatient service, and after the identification of skin lesions for children managed in the neurology service. Comparison between these diagnostic markers for medical outpatients compared with neurology outpatients demonstrated a trend, but it was not statistically significant on Fisher's exact test $(p=0.058)$.

Prior to 2007, the median value for the number of new children with TSC who were referred to the service was 1 per year; from 2007, this number increased to a median value of 3 per year.

\section{Discussion}

This study illustrated that awareness of TSC in SA health services is poor. TSC was suspected in only a few children, based on the presence of skin lesions; their referral to the neurology service was typically because of recurrent seizures. Most of the remaining children presented after acute presentations with seizures, and the diagnosis of TSC was made following admission for emergency management. Children in our cohort presented later than those reported in international studies (Table 1). As found in our study and in large international studies, the presenting features of TSC can be diverse - prior to their diagnosis a proportion of our children were under direct management in the genetics, gastroenterology and dermatology services. ${ }^{[4]}$ The skin manifestations of 2 of the patients were detected, but incorrectly interpreted. In settings where conditions such as neurocysticercosis are prevalent, erroneous assumptive diagnoses based on neuroimaging findings may also occur, as illustrated in this study.

Hypomelanocytic macules may be present at birth, and other skin manifestations such as facial angiofibroma and shagreen patches appear later during the first decade of life. ${ }^{[2]}$ In this study, even though hypomelanocytic macules were found in $69 \%$ of patients, the diagnosis of TSC was suspected at referral in only $33 \%$ of cases (Table 1).

The 2012 international guidelines on the diagnosis and management of TSC emphasise the importance of early diagnosis to improve the outcome of affected children. ${ }^{[1]}$ Epileptic spasms are common during the first year of life in children with TSC. These children may also have focal seizures, which may occur either before or after onset of epileptic spasms. Corticosteroids are the recommended treatment for infants with epileptic spasms who do not have TSC, while vigabatrin is the most effective intervention in infants with TSC. ${ }^{[8]}$ Delayed recognition of TSC can lead to suboptimal intervention for epileptic encephalopathy. The guidelines recommend presymptomatic electroencephalography (EEG) in the age group at risk of epileptic spasms. ${ }^{[2,5]}$ 
While this is a viable intervention at our centre, patients in this study typically had already evolved seizures as a presenting complaint. Furthermore, EEG is a scarce resource in low- and low-middleincome countries in Africa and many centres struggle to access such testing. Epileptic spasms were identified in $18 \%$ of the children in our study, which is similar to the percentage in other studies. ${ }^{[9]}$

The guidelines have similar recommendations for renal monitoring to screen for angiomyolipomas. Even though this is also a rational recommendation, it is unlikely to be fullfilled in many parts of subSaharan Africa. ${ }^{[1,2,10]}$ In our group, 3 patients presented with cardiac rhabdomyomas, and 5 had lesions identified after TSC was diagnosed. It is possible that these additional cases reflected limited antenatal assessment, and further highlights the difficulties of early detection in our setting. ${ }^{[1]}$ The challenges illustrated here highlight the need for these children to be managed in a tertiary setting, where adequate multidisciplinary care can be provided, and the drain on already stretched secondary and primary healthcare services can be avoided.

The study by Yates et al. ${ }^{[5]}$ reported similar presentation triggers and phenotypic features as in our cohort, but in a population in the UK. One hundred and fourteen children in this study met the criteria for TSC and $17 \%$ were diagnosed in the antenatal period, compared with $8 \%$ of our patients. ${ }^{[5]}$ Thirty children $(24 \%)$ in the UK study presented before the onset of seizures, which affected $77(62 \%)$ of the group. ${ }^{[5]}$ In our population, the diagnosis of TSC before neuroimaging for new onset of seizures occurred less often than in the Yates et al. ${ }^{[5]}$ study, but the proportion of children presenting with seizures was comparable (62\% in the UK study v. $56 \%$ in the current study). The UK study also found an increasing number of children presenting prenatally or in early infancy, which reflected both the increased awareness of TSC and appropriate resources available to identify affected patients. ${ }^{[5]}$

The international TSC guidelines also recommend early neurodevelopmental screening to optimise performance outcomes. This would permit appropriate school placement and learning support to be initiated. There are challenges to such implementation in our setting, especially where there are additional compounding factors of poor socioeconomic structure, and layering effects from other comorbidities, such as HIV and tuberculosis. In most parts of Africa, there is limited access to developmental intervention, with few skilled health practitioners and long waiting lists.

Subependymal giant cell astrocytomas (SEGAs) are some of the more serious complications of TSC. International guidelines recommend regular monitoring and awareness of early symptoms and signs to facilitate early intervention. ${ }^{[1]}$ The current study found a higher proportion of children with SEGAs compared with the UK, USA and Turkey. ${ }^{[5-7]}$ This may reflect disease behaviour, whereby only the most symptomatic patients access healthcare services in our setting. In SA, there is no effective structure for routine screening of asymptomatic children for TSC.

Genetic testing is useful to confirm the diagnosis of TSC and for family counselling. ${ }^{[5]}$ Currently, genetic testing is not routinely available in SA and fulfilment of clinical criteria is used to confirm a diagnosis of TSC. However, the service does have access to genetic counsellors, who provide families with support. A previous study ${ }^{[12]}$ showed that caregivers, who despite regular attendance at the clinic, often failed to understand the condition and benefited when provided with written information, preferably in their home language or language of choice. This study highlights that there is a great need to raise awareness of TSC in our setting. Based on service referral patterns, opportunities to achieve this could be by promoting TSC teaching in undergraduate curricula, in community projects, and by raising awareness in primary healthcare clinics. Informative posters could be placed in strategic settings, such as primary healthcare clinics and community centres. In SA, a national TSC non-governmental organization is yet to be established, but there are other routes of support, such as Rare Diseases SA (http://rarediseases.co.za/) and Epilepsy SA (http://epilepsy.org.za).

Many of the patients were incidentally diagnosed by neuroimaging performed as part of evaluation of their seizure disorder. In our setting, neuroimaging is not usually performed unless there is concern of focal pathology. Further, in our experience seizures are often erroneously assumed to be generalised, also reducing the likelihood of performing neuroimaging. This highlights the need for extracting a good clinical seizure semiology and performing a careful clinical examination, as the majority of affected children have skin manifestations. Since 2007, the RCWMCH TSC service has actively promoted awareness of common clinical markers for TSC within the hospital structure - a tertiary care facility. The median annual referral rate has tripled. While this is a positive trend, heightened awareness needs to be facilitated at primary and secondary facilities as the next stage.

Based on the apparent scarcity of recognition and limited awareness of TSC in SA, which, compared with other parts of Africa, has greater access to resources, it is likely that the challenges of diagnosing TSC are even more pronounced in the rest of the continent. The late diagnosis of TSC in adulthood is associated with greater complications. ${ }^{[13]}$

\section{Conclusion}

In this study, most children with TSC presented as emergency cases with seizures. Awareness of the common clinical markers at presentation is lacking. We aimed to gain insight into the referral patterns of children with TSC to a specialised service to identify ways to improve early identification of and intervention with regard to the condition. The next stage will be an intervention study to observe if the recruitment can be improved by raising awareness in the key locations where these children present, inclusive of primary healthcare clinics, community bases, and school healthcare clinics.

TSC prevalence is estimated internationally at 1 in 6000 newborns, and based on our population of 2.14 million children $<19$ years of age in the Western Cape, some 357 children in this region may have TSC. ${ }^{[14]}$ The majority of affected children in our area and potentially sub-Saharan Africa are probably not diagnosed. This highlights the need to raise awareness of such common clinical markers to promote early referral to specialist centres to reduce complications.

Acknowledgements. The authors would like to thank the patients, parents and carers for agreeing to participate in this study.

Author contributions. EK, MW and PS assisted with the data collection; EK drafted the manuscript; BS identified patients for study inclusion, assisted with consent and contributed to the manuscript content; and JW designed and conceived the study and revised the final manuscript.

Conflict of interest. JW and BS are members of the SA Tuberous Sclerosis Complex advisory committee, which was established with funding support from Novartis.

1. Krueger DA, Northrup H. International Tuberous Sclerosis Complex Consensus G. Tuberous sclerosis complex surveillance and management: Recommendations of the 2012 International Tuberous Sclerosis Complex Consensus Conference. Pediatr Neurol 2013;49(4):255-265. https://doi.org/10.1016/j. pediatrneurol.2013.08.002

2. Northrup H, Krueger DA, Group ITSCC. Tuberous sclerosis complex diagnostic criteria update: Northrup H, Krueger DA, Group ITSCC. Tuberous sclerosis complex diagnostic criteria update
Recommendations of the 2012 International Tuberous Sclerosis Complex Consensus Conference. Pediatr Neurol 2013;49(4):243-254. https://doi.org/10.1016/j.pediatrneurol.2013.08.001 
3. Rentz AM, Skalicky AM, Liu Z, et al. Tuberous sclerosis complex: A survey of health care resource use and health burden. Pediatr Neurol 2015;52(4):435-441. https://doi.org/10.1016/j.pediatrneurol.2014.11.013

4. Kothare SV, Singh K, Hochman T, et al. Genotype/phenotype in tuberous sclerosis complex: Associations with clinical and radiologic manifestations. Epilepsia 2014;55(7):1020-1024. https://doi.org/10.1111/ epi. 12627

5. Yates JR, Maclean C, Higgins JN, et al. The Tuberous Sclerosis 2000 Study: Presentation, initial assessments and implications for diagnosis and management. Arch Dis Child 2011;96(11):1020-1025. assessments and implications for diagnosis
https://doi.org/10.1136/adc.2011.211995

6. Datta AN, Hahn CD, Sahin M. Clinical presentation and diagnosis of tuberous sclerosis complex in infancy. J Child Neurol 2008;23(3):268-273. https://doi.org/10.1177/0883073807309250

7. Erol İ, Savaș T, Şekerci S, et al. Tuberous sclerosis complex: Single center experience. Turk Pediatri Ars 2015;50(1):51-60.

8. Friedman D, Bogner M, Parker-Menzer K, Devinsky O. Vigabatrin for partial-onset seizure treatmen in patients with tuberous sclerosis complex. Epilep Behav 2013;27(1):118-120. https://doi.org/10.1016/j. yebeh.2012.12.033

9. Kothare SV, Singh K, Chalifoux JR, et al. Severity of manifestations in tuberous sclerosis complex in relation to genotype. Epilepsia 2014:55(7):1025-1029. https://doi.org/10.1111/epi.12680
10. Ouzaid I, Autorino R, Fatica R, et al. Active surveillance for renal angiomyolipoma: Outcomes and factors predictive of delayed intervention. BJU Int 2014;114(3):412-417, https://doi.org/10.1111/bju.12604 1. Shen $\mathrm{Q}$ Shen J Qino Z Yao Q Huang G, Hu X. Cardiac rhabdomyomas associated with tuberous sclerosis complex in children: From presentation to outcome. Herz 2015;40(4):675-678. https://doi. sclerosis complex in children:

12. Samia P, Donald KA, Schlegel B, Wilmshurst JM. Parental understanding of tuberous sclerosis complex. J Child Neurol 2015;30(10):1281-1286. https://doi.org/10.1177/0883073814558121

13. Seibert D, Hong CH, Takeuchi F, et al. Recognition of tuberous sclerosis in adult women: Delayed 3. Seibert D, Hong CH, Takeuchi F, et al. Recognition of tuberous sclerosis in adult women: Delayed
presentation with life-threatening consequences. Ann Intern Med 2011;154(12):806-813. https://doi.
org/10.7326/0003-4819-154-12-201106210-00008 org/10.7326/0003 14. Statistics South Africa. 2014. http://statssa.gov.za/publications/P0302/P03022014 (accessed 19 January
2017).

Accepted 7 December 2016 